\title{
Pyoderma Gangrenosum Overlying Venous Insufficiency: An Inevitable Misdiagnosis
}

\author{
Jonghyun Park ${ }^{1}$, Guk Jin Jeong ${ }^{2}$, Ji Yeon Hong ${ }^{2}$, Kui Young Park², Beom Joon Kim², Woo Seob Kim¹ \\ Departments of ${ }^{1}$ Plastic and Reconstructive Surgery and ${ }^{2}$ Dermatology, Chung-Ang University College of Medicine, Seoul, Korea
}

\begin{abstract}
Pyoderma gangrenosum (PG) is a destructive inflammatory disease characterized by painful ulcerative lesions with neutrophilic infiltration in the dermis. Although the etiology and pathogenesis are poorly understood, uncontrolled inflammatory responses to nonspecific stimuli may trigger the development of PG. Here, we report a rare case of PG arising above a venous insufficiency lesion. The most typical diagnosis for an ulcer at such a location is venous ulcer. However, our atypical case of PG emphasizes that PG can be induced by ischemic conditions, such as venous insufficiency. Debridement and skin grafting, which were performed based on an incorrect diagnosis, worsened the PG lesion and provoked a severe pathergy phenomenon. Thus, surgeons should consider PG in the differential diagnosis of intractable venous ulcers in order to ensure an accurate diagnosis and prompt treatment.
\end{abstract}

Keywords: Pyoderma gangrenosum; Venous insufficiency; Venous ulcer

\section{Introduction}

Pyoderma gangrenosum (PG) is a destructive inflammatory disease characterized by painful ulcerative lesions with neutrophilic infiltration of the dermis. The etiology and pathogenesis of PG are poorly understood [1]. The pathergy phenomenon, a hallmark of PG, is a condition in which trivial trauma leads to the development of similar lesions that may be resistant to healing [2]. This phenomenon implies that uncontrolled inflammatory responses to nonspecific stimuli may trigger PG [3]. Approximately $50 \%$ of PG cases have been associated with a wide variety of disorders, including inflammatory bowel disease and other hematologic and rheumatologic diseases [4]. However, few cases of PG have developed after only minor trauma with ischemia $[5,6]$. Herein, we presented the case of 66-year-old man, who developed PG masquerading intractable venous ulcers. The written informed consent was obtained from the patient.

\section{Case}

A 66-year-old man was referred to Chung-Ang University Hospital and presented with multiple painful ulcers on both lower legs. These ulcers had been recurrent over the past 4 years. A year before the ulcers arose, he had undergone a total knee replacement surgery (TKR) for degenerative osteoarthritis. The ulcers were well circumscribed, with purplish elevated borders, and their granulomatous bases were covered with yellow fibrinous tissue. Pitting edema surrounded by a brown, reticular pigmented patch was also identified (Fig. 1). Before being referred to our clinic, he had \\ Case Report \\ Received: February 15, 2019 \\ Revised: February 23, 2019 \\ Accepted: February 25, 2019

\section{Corresponding author: Woo Seob Kim, M.D., Ph.D.} \\ Department of Plastic and Reconstructive \\ Surgery, Chung-Ang University Hospital, \\ Chung-Ang University College of Medicine, 102 \\ Heukseok-ro, Dongjak-gu, Seoul 06973, Korea \\ Tel: +82-2-6299-1615 \\ Fax: +82-2-6299-1718 \\ E-mail: kimws@cau.ac.kr
}

This is an Open Access article distributed under the terms of the Creative Commons Attribution Non-Commercial License (http://creativecommons.org/licenses/by-nc/4.0/) which permits unrestricted non-commercial use, distribution, and reproduction in any medium, provided the original work is properly cited.

(c) 2019 Korean Wound Management Society 
been diagnosed with a venous leg ulcer based on clinical appearance and history of TKR, and he was treated with conventional therapy, including leg elevation, wound dressing, oral anticoagulants, antibiotics, and five split-thickness skin grafts. Despite the prolonged treatment of the venous leg ulcers, clinical improvement was not observed, and new lesions developed at the skin graft donor and recipient sites. These new lesions on donor sites demonstrated a pathergy phenomenon. For further evaluation, an immunological assessment was carried out: the presence of anti-cardiolipin antibody, rheumatoid factor, lupus anticoagulant, anti-double-stranded DNA antibody, anti-Ro/SS-A, and anti-La/SS-B was tested. The results of these tests were negative. A biopsy from the center of the lesion revealed diffuse neutrophilic infiltration in the necrotic upper dermis and neutrophil-predominant perivascular infiltration with minimal vascular damage. We did not find any fi- brinous deposits or intravascular thrombosis (Fig. 2). Because of the combination of certain characteristics, such as pathergy and neutrophilic infiltrates on biopsy, a final diagnosis of PG was made after excluding other diseases such as malignancy and infections. Therefore, treatment with high-dose systemic corticosteroids ( $45 \mathrm{mg} /$ day) was initiated along with conventional treatment for venous ulcers, including leg elevation and dressing changes. During the follow-up period, the lesion resolved dramatically, and his pain rapidly diminished (Fig. 3).

\section{Discussion}

The mechanisms and pathophysiology of PG are elusive and likely multifactorial. An accepted and validated diagnostic criterion for PG is still lacking [7]. An anomalous autoimmune or inflammatory response is believed to be the main compo-
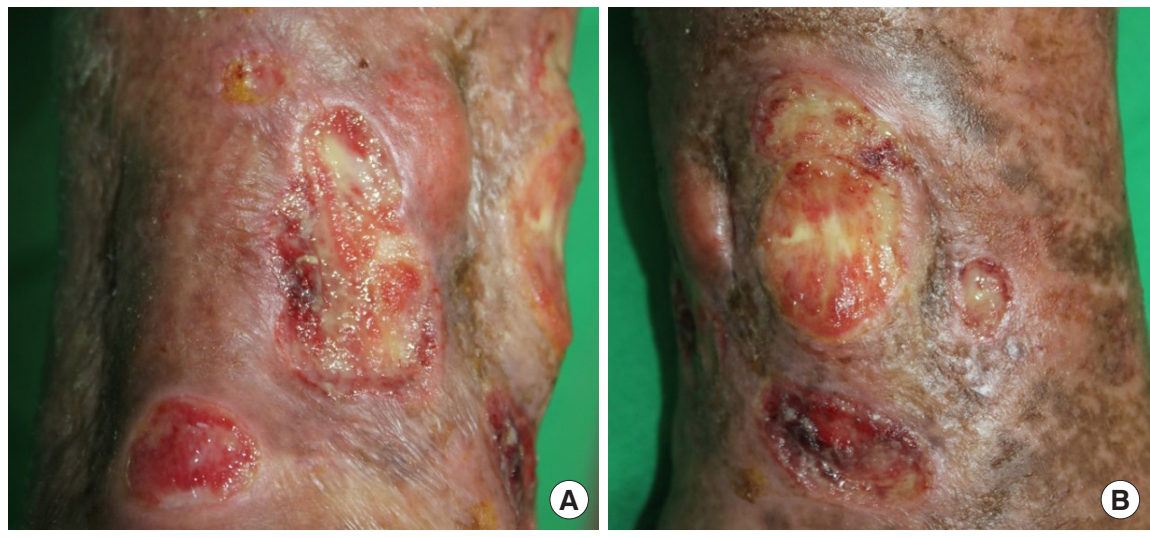

Fig. 1. Multiple necrotic ulcers with purplish, elevated borders. (A, B) Leg edema surrounded by a brownish, reticular pigmented patch was also present.
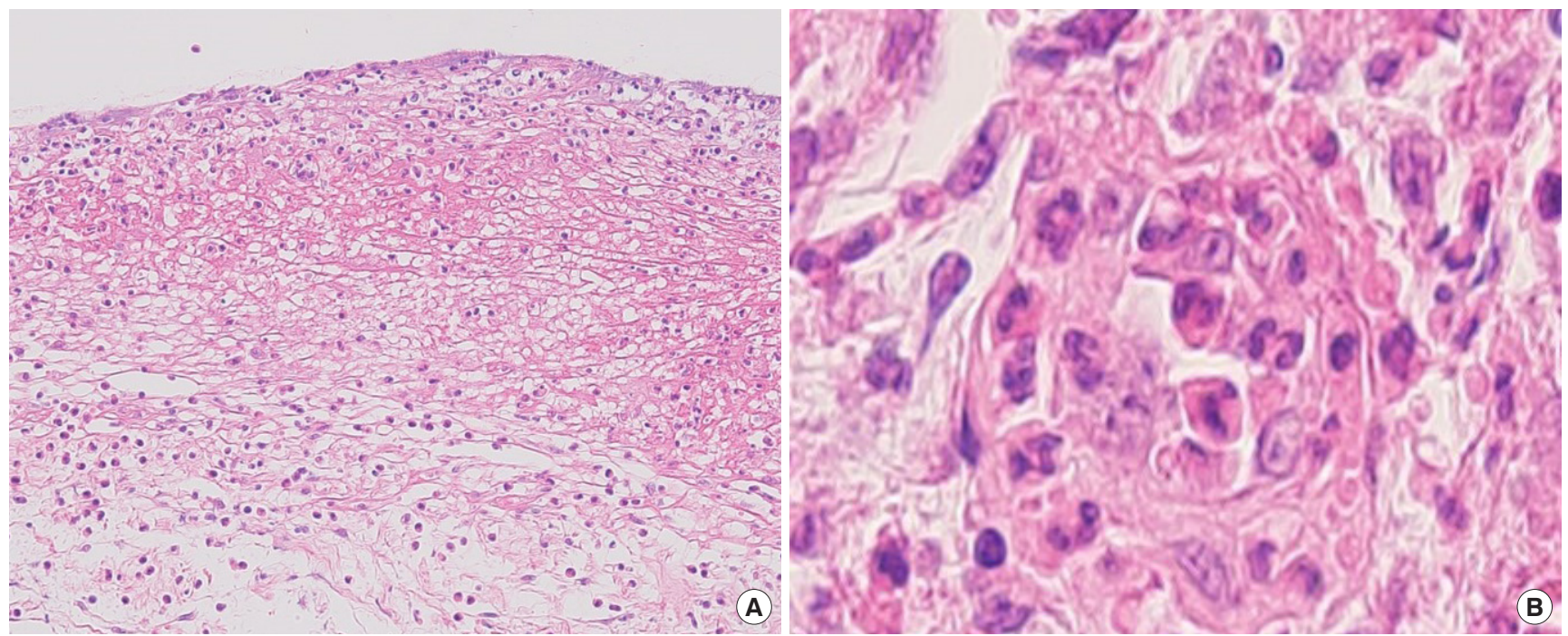

Fig. 2. Biopsy of the ulcer. (A) Diffuse neutrophilic infiltration in the necrotic upper dermis (H\&E stain, $\times 100)$. (B) Neutrophil-predominant perivascular infiltration with minimal vascular damage (H\&E stain, $\times 400)$. 

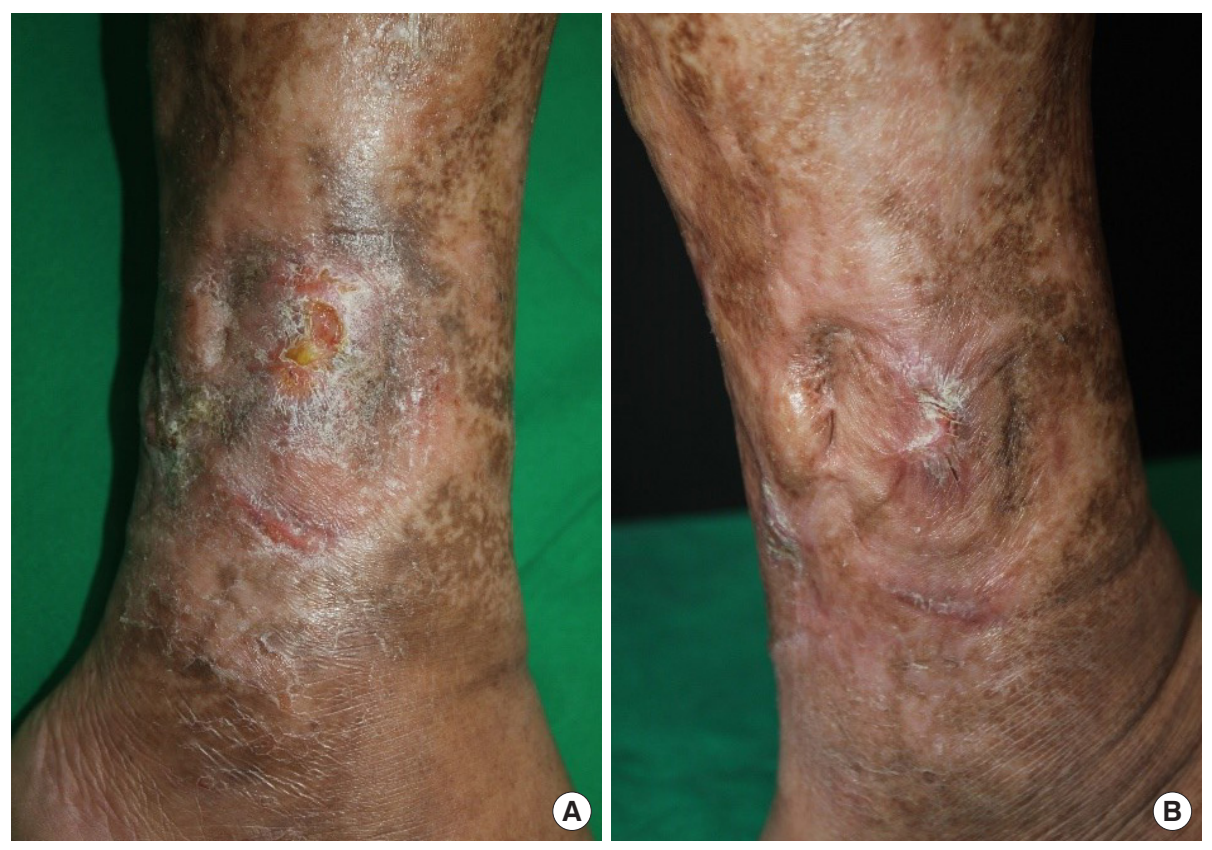

Fig. 3. Results after the treatment. (A) Two months and (B) 4 months after steroidal treatment.

nent of PG's pathophysiology [8], and this is supported by the efficacy of immunosuppression with systemic steroids and cyclosporine in treating PG. Furthermore, inflammatory and autoimmune responses are exacerbated in ischemic conditions such as venous insufficiency [9].

Su et al. [10] proposed diagnostic criteria for PG as fulfilling two of major criteria and two of minor criteria of the following. Major criteria: rapid progression of a painful, necrolytic cutaneous ulcer with an irregular, violaceous border, and exclusion of other causes of cutaneous ulceration. Minor criteria: history suggestive of pathergy or clinical finding of cribriform scarring, systemic diseases associated with PG, compatible histopathological findings and response to treatment. In our case, though three of the minor criteria was fulfilled except for having systemic diseases, major criteria was not fulfilled at first for excluding other causes of ulceration was not possible because of the chronic venous ulcer patient had previously. In addition, Koo et al. [11] suggested some of the characteristics that could differentiate PG from chronic venous ulcer. While chronic venous ulcer usually affect only legs, PG could appear on different parts of the body and also is usually associated with systemic diseases and pathergy with distinct features of pustules, purulent discharge, undermined borders, and craterlike holes. However, in our case, ulceration only appeared on legs and did not seem to present the features that differentiated PG from venous ulcer.
We found our case fascinating because the lesion did not quite show known characteristics of PG and plus was superimposed over a site of venous insufficiency, confusing clinicians and leading to an inevitable misdiagnosis of venous ulcers, thus delaying appropriate treatment. The debridement and skin grafting, which were performed based on an incorrect diagnosis, worsened the PG lesion and provoked a severe pathergy phenomenon. This led us into immunological assessments and skin biopsy to rule out other possible diseases and concluded with an elusive PG and properly treat the patient.

In conclusion, to avoid misdiagnosis, a skin biopsy should be considered when a venous leg ulcer is resistant to healing with conventional ulcer treatments. Like in our case, though the featuring characteristics of the lesion may not let clinicians suspect a disease, biopsy could be a key step in diagnosing a hard case. Biopsies are useful, not only in diagnosis of PG, but also in excluding other diseases such as malignancy and infections [12]. Taking variety of diagnosing tools into consideration for chronic wounds will not just save time, but will have a major effect on the outcome of the treatment.

\section{Conflict of interest}

No potential conflicts of interest relevant to this article are reported. 


\section{Acknowledgments}

Jonghyun Park https://orcid.org/0000-0001-9910-6945

Guk Jin Jeong https://orcid.org/0000-0002-2379-0370

Ji Yeon Hong https://orcid.org/0000-0002-6935-7118

Kui Young Park https://orcid.org/0000-0001-5965-1754

Beom Joon Kim https://orcid.org/0000-0003-2320-7621

Woo Seob Kim https://orcid.org/0000-0002-4104-3926

\section{References}

1. Amin SV, Bajapai N, Pai A, et al. Pyoderma gangrenosum in two successive pregnancies complicating caesarean wound. Case Rep Obstet Gynecol 2014;2014:654843.

2. Yasin F, Assad S, Zahid M, et al. Extensive pyoderma gangrenosum: a challenging diagnosis and literature review of management. Cureus 2017;9:e1486.

3. Rogge FJ, Pacifico M, Kang N. Treatment of pyoderma gangrenosum with the anti-TNFalpha drug - Etanercept. J Plast Reconstr Aesthet Surg 2008;61:431-3.

4. Teagle A, Hargest R. Management of pyoderma gangreno- sum. J R Soc Med 2014;107:228-36.

5. Hadid W, Patel P, Piette WW. After the injury. Am J Med 2007;120:140-2.

6. Keskin M, Tosun Z, Ucar C, et al. Pyoderma gangrenosum in a battered child. Ann Plast Surg 2006;57:228-30.

7. Alavi A, French LE, Davis MD, et al. Pyoderma gangrenosum: an update on pathophysiology, diagnosis and treatment. Am J Clin Dermatol 2017;18:355-72.

8. Wollina U, Haroske G. Pyoderma gangraenosum. Curr Opin Rheumatol 2011;23:50-6.

9. Liao YH, Cheng X. Autoimmunity in myocardial infarction. Int J Cardiol 2006;112:21-6.

10. Su WP, Davis MD, Weenig RH, et al. Pyoderma gangrenosum: clinicopathologic correlation and proposed diagnostic criteria. Int J Dermatol 2004;43:790-800.

11. Koo K, Brem H, Lebwohl M. Pyoderma gangrenosum versus chronic venous ulceration: comparison of diagnostic features. J Cutan Med Surg 2006;10:26-30.

12. Cranendonk DR, Lavrijsen APM, Prins JM, et al. Cellulitis: current insights into pathophysiology and clinical management. Neth J Med 2017;75:366-78. 